\title{
A PET/CT-based Morphometric Study of Spinal Canal in Korean Young Adults: Anteroposterior Diameter from Cervical Vertebra to Sacrum
}

\author{
Moo Sung Kang, Jeong Yoon Park, Dong Kyu Chin, Kyung Hyun Kim, \\ Sung Uk Kuh, Keun Su Kim, Yong Eun Cho \\ Department of Neurosurgery, Spine and Spinal Cord Institute, Gangnam Severance Hospital, \\ Yonsei University College of Medicine, Seoul, Korea
}

\begin{abstract}
Objective: To establish normative data for spinal canal AP diameter from cervical vertebra to sacrum in the Korean young and to assess the exposed spinal canal after laminectomy which was related with restenosis by post-laminectomy membrane formation.

Methods: From PET/CT, axial bone-window CT of 83 young adults (20-29 years) were obtained, and we measured AP diameters of C3, C5, C7, T1, T4, T8, T12, L1, L3, L5 and S1. We also measured exposed AP diameter of C3, C5, C7, T1 and T2 above imaginary line for laminectomy.

Results: The shortest mean AP diameter was at C5 $(14.5 \pm 1.5 \mathrm{~mm})$, and the longest was at $\mathrm{S} 1(17.4 \pm 2.3 \mathrm{~mm})$. AP diameter increased from C3 $(14.6 \pm 1.1 \mathrm{~mm})$ to $\mathrm{T} 1(16.1 \pm 1.2 \mathrm{~mm})$ at cervical spine. In the thoracic spine, the diameter gradually decreased from T1 $(16.1 \pm 1.2 \mathrm{~mm})$ to T8 $(14.6 \pm 1.3 \mathrm{~mm})$ and increased to T12 $(16.7 \pm 1.2 \mathrm{~mm})$. The diameter decreased from L1 $(16.7 \pm$ $1.3 \mathrm{~mm})$ to $L 3(15.7 \pm 1.9 \mathrm{~mm})$, and it increased to $S 1(17.4 \pm 2.3 \mathrm{~mm})$ at lumbar spine. Exposed AP diameter above imaginary line for laminectomy was the longest at C3 $(4.8 \pm 1.2 \mathrm{~mm})$ and gradually decreased to T1 $(3.3 \pm 0.9 \mathrm{~mm})$ and T2 $(0 \mathrm{~mm})$. Conclusions: Spinal AP diameter was the shortest in the mid-cervical area (C5) and increased to the upper thoracic area. From the upper thoracic vertebra, the diameter gradually decreased to the mid-thoracic vertebra (T8) and then increased to the lower thoracic vertebra. Lumbar vertebra also was similar with thoracic vertebra. Below T2, there was no exposed dural sac after laminectomy. This means that restenosis by post-laminectomy membrane formation can occur above T1.
\end{abstract}

Key Words: Spinal canal $\cdot$ Cervical vertebrae $\cdot$ Thoracic vertebrae $\cdot$ Lumbar vertebrae

\section{INTRODUCTION}

Accurate anatomic descriptions of vertebral anatomy are necessary for the diagnosis of various spinal diseases. Several previous studies have investigated the morphometry of the vertebrae using different experimental techniques such as direct measurements, roentgenography with plain films, CT, and $\mathrm{MRI}^{2,7}$, 13-15,19,21). However, these studies focused on selected areas such as cervical, thoracic, or lumbar area. No study has examined the whole spine from cervical to sacrum. In addition, these

- Received: May 3, 2012 - Revised: September 11, 2012

- Accepted: September 25, 2012

Corresponding Author: Jeong Yoon Park, MD

Department of Neurosurgery, Gangnam Severance Hospital, 211, Eonju-ro

Gangnam-gu, Seoul 135-720, Korea

Tel: +82-2-2019-3390, Fax: +82-2-3461-9229

Email: spinepjy@gmail.com studies included various age groups from children ${ }^{20)}$ to old age $^{7,14)}$. It is very difficult to know the normal anatomic characteristics of adults in whom growth is complete and no degenerative change has occurred.

Because of recent development of spinal instruments, recent anatomical studies focused on the pedicle instead of the spinal canal $^{5,13)}$. The spinal canal is as important as the pedicle in anatomical study. The cross-sectional areas of the spinal canal are of clinical importance in traumatic, degenerative, and inflammatory conditions, and a small spinal canal diameter has been associated with an increased risk of injury ${ }^{15)}$. Actually it is difficult to measure cross-sectional areas of the spinal canal, because spinal canal has various shapes. Thus, we measured spinal canal anteroposterior (AP) diameters instead of the crosssectional areas.

The purpose of this study was to establish normative data for spinal canal AP diameters from the cervical vertebra to sacrum in the Korean young adults without degenerative 
change. We postulated that this study could provide a useful set of reference sizes of the normal spinal canal in Asian and Korean. In addition, we assessed the exposed spinal canal after laminectomy. Laminectomy for multilevel cervical myelopathy has been avoided because of progressive kyphosis, perineural adhesions and restenosis by post-laminectomy membrane formation ${ }^{4,10,17)}$. A restenosis by post-laminectomy membrane formation associated with exposed dural sac after laminectomy $^{4)}$. To examine the influence of post-laminectomy membrane formation, we also investigated the exposed spinal canal after imaginary removal of the lamina from cervical to thoracic vertebra.

\section{MATERIALS AND METHODS}

\section{Patients}

This study was approved by the institutional review board of Gangnam Severance Hospital, Yonsei University College of Medicine (No 3-2011-0111); however, patient consent was not required for the retrospective review of records and images because patient anonymity was preserved. From January 2005 to January 2010, young adult patients (age: 20-29 years) underwent Positron emission tomography (PET)/computed tomography (CT) (Biograph-64 True Point, Siemens, Erlangen, Germany), to evaluate for meta- stasis after diagnosed various cancer. Over 150 whole spine data were available for the study. Exclusion criteria were a history or PET/CT diagnosed cancer, tumor or mass on the spine and the nervous system, spinal abnormality, autoimmune disease, connective tissue disease, congenial anomaly, inflammation, infection, and a previous surgery on the spine.

\section{Evaluation of Axial bone-window CT images from PET/CT}

We obtained PET/CT data of 83 young adults, which were obtained via a Siemens Biograph-64 True Point PET/CT. Axial bone-window CT images were obtained using PET/CT, and PACS software and a PACS workstation (Centricity 2.0, General Electrics Medical Systems, Milwaukee, WI) were used to check spinal canal AP diameters at the mid-vertebral body level by an independent spine surgeon and a neuroradiologist. $3 \mathrm{~mm}$ cutting of PET/CT was done in perpendicular to the table, and reformatted axial images parallel to disc space were made. When CT was done in perpendicular to table, sometimes we obtained oblique axial views of the spinal canal, and oblique views were longer than the real AP diameter, so we made reformatted axial images parallel to disc space. From reformatted vertebral body axial images parallel to the disc space, reconstructed whole spine sagittal image was made (Fig. 1). AP spinal canal diameters of cervical vertebrae 3, 5, 7 (C3, 5 , 7), thoracic vertebrae $1,4,8,12(\mathrm{~T} 1,4,8,12)$, lumbar vertebrae 1, 3, 5 (L1, 3, 5) and sacrum 1 (S1) were measured by axial image parallel to disc space at mid-vertebral body part (Fig. $1 \mathrm{~B}$ to D).

To examine the influence of post-laminectomy membrane formation, we drew an imaginary line between facet joint and lamina at $\mathrm{C} 3, \mathrm{C} 5, \mathrm{C} 7$, and $\mathrm{T} 1$, we also measured the AP diameter of the spinal canal areas above imaginary line, which will

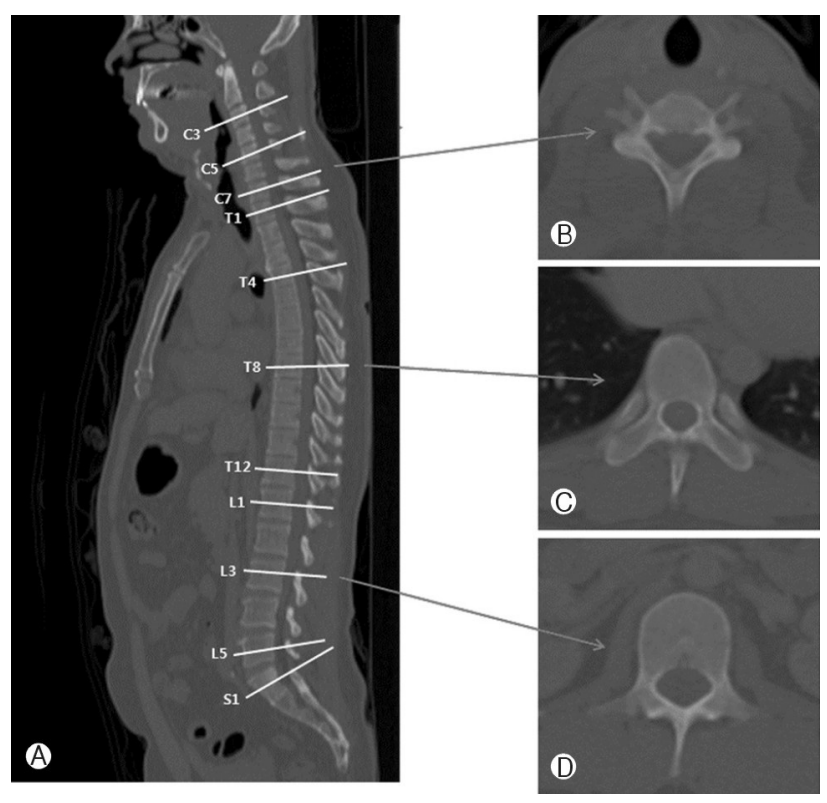

Fig. 1. From vertebral body axial images parallel to the disc space, reconstructed whole spine sagittal image $(A)$ was made. AP spinal canal diameters of cervical vertebrae 3, 5, 7 (B), thoracic vertebrae 1, 4, 8, $12(\mathrm{C}$ ), lumbar vertebrae 1, 3, 5 (D) and sacrum 1 were measured by axial image parallel to disc space at mid-vertebral body part .
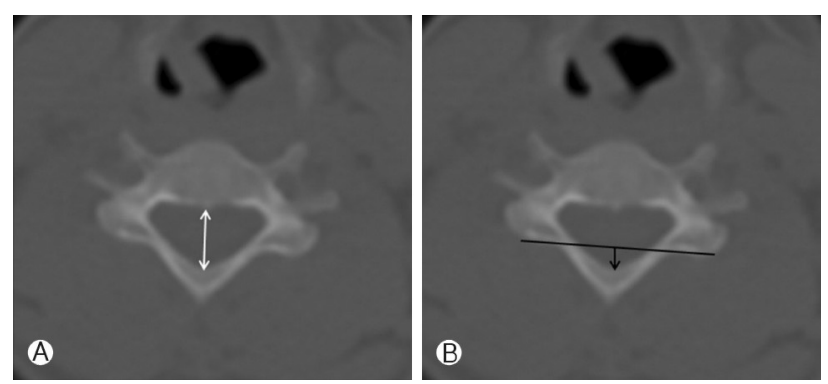

Fig. 2. Mid-vertebral body bone-window CT images, C5 were obtained from PET/CT. The white arrow indicates the spinal canal AP diameter. The black line indicates an imaginary laminectomy line between lateral mass and lamina. The black arrow indicates the exposed spinal canal AP diameter after laminectomy. 
be exposed after laminectomy (B of Fig. 2 and 3). Because there was no spinal canal exposure above imaginary laminectomy line, we did not measured exposed AP diameter of spinal canal above imaginary line below T1 (Fig 4, 5).

With three independent doctor's results, the authors calculated average of all numerical values. SPSS for Windows (version 15.0 K; SPSS, Chicago, IL) was used for the analysis. Data of women and men were compared using the paired t-test. p-values of $<0.05$ were considered significant. There was no external funding source for the present study.

\section{RESULTS}

The average spinal canal AP diameter at each level and according to gender is shown in Table 1 . The shortest mean
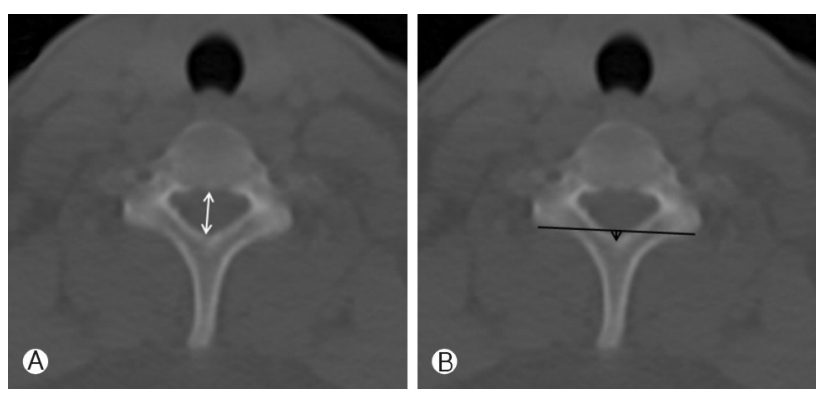

Fig. 3. Mid-vertebral body bone-window CT images, $\mathrm{Tl}$ were obtained from PET/CT.
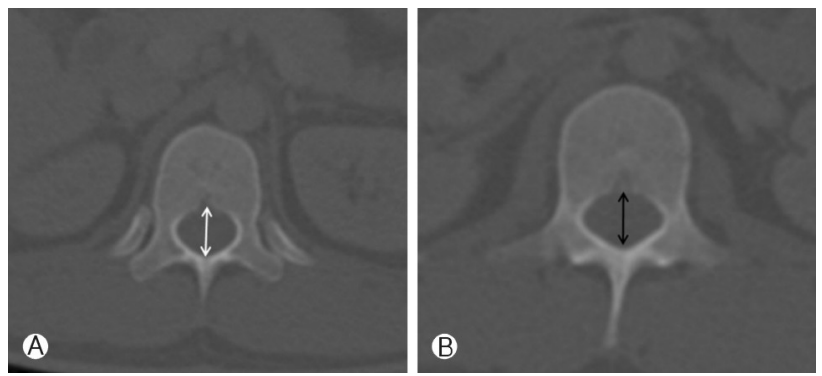

Fig. 4. Mid-vertebral body bone-window CT images of T12, L1 were obtained from PET/CT. The white arrow indicates the spinal canal AP diameter of T12 (A), L1 (B).

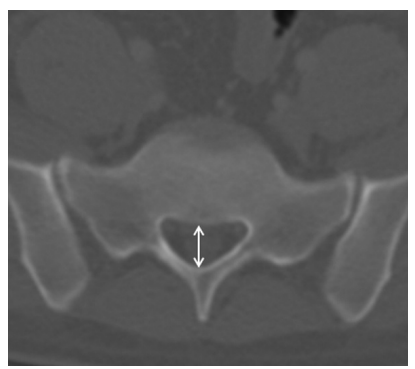

Fig. 5. Mid-vertebral body bonewindow $\mathrm{CT}$ images of $\mathrm{S} 1$ were obtained from PET/CT.
AP diameter was at C5 $(14.5 \pm 1.5 \mathrm{~mm})$, and the longest was at $S 1(17.4 \pm 2.3 \mathrm{~mm}$ ) (Table 1) (Fig. 6). AP diameter increased from C3 $(14.6 \pm 1.1 \mathrm{~mm})$ to $\mathrm{T} 1(16.1 \pm 1.2 \mathrm{~mm})$ in the cervical spine (Fig. 6). In the thoracic spine, the diameter gradually decreased from T1 $(16.1 \pm 1.2 \mathrm{~mm})$ to T8 $(14.6 \pm 1.3 \mathrm{~mm})$ and increased to T12 (16.7 $\pm 1.2 \mathrm{~mm})$ (Fig. 6). From L1 (16.7 \pm 1.3 $\mathrm{mm})$ to $\mathrm{L} 3(15.7 \pm 1.9 \mathrm{~mm})$, the diameter decreased and then increased to S1 (17.4 $\pm 2.3 \mathrm{~mm}$ ) (Fig. 6). The measurements of the diameters were not significantly different between women and men ( $\mathrm{p}>0.05)$ (Table 1).

The diameter of the AP spinal canal to be exposed after laminectomy above the imaginary laminectomy line was the longest at C3 $(4.8 \pm 1.2 \mathrm{~mm})$ and gradually decreased from $\mathrm{T} 1$ $(3.3 \pm 0.9 \mathrm{~mm})$ to T2 $(0 \mathrm{~mm})$ (Table 1 and Fig. 7). Below T1, there was no exposed spinal canal area after laminectomy above the imaginary line (Fig. 7). The ratio of exposed AP diameter to spinal canal AP diameter was also largest at C3 (32.9 \%) (Fig. 8).

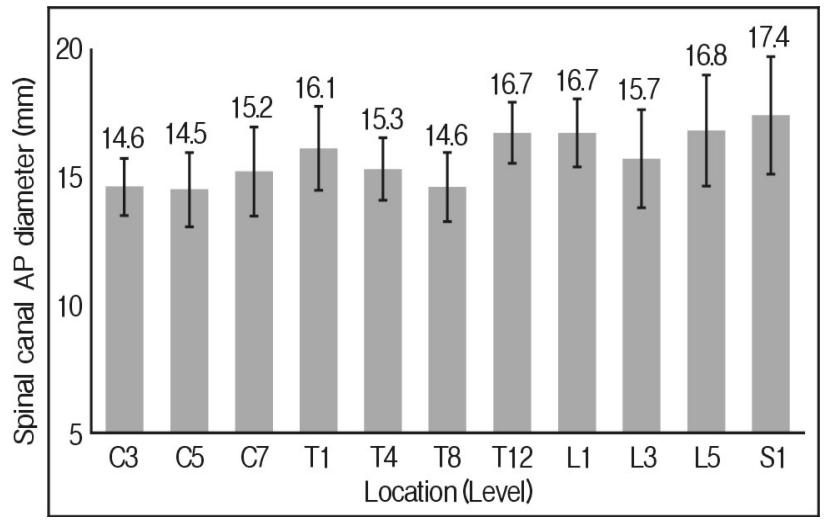

Fig. 6. Spinal canal AP diameter from C3 to S1.

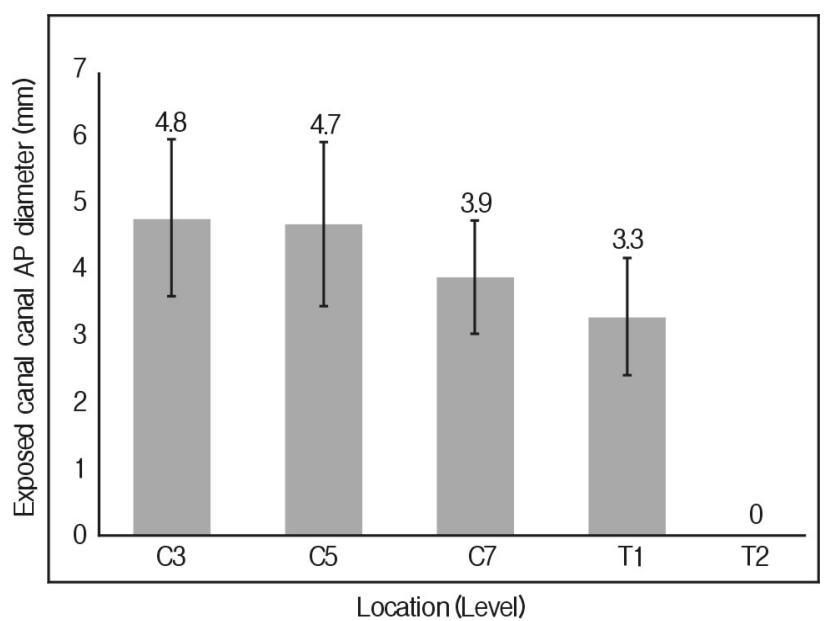

Fig. 7. Exposed spinal canal AP diameter above imaginary laminectomy line between facet joint and lamina, from C3 to T2. 
Table 1. Spinal canal anteroposterior diameter at each level according to gender and after laminectomy

\begin{tabular}{|c|c|c|c|c|c|c|c|c|c|c|c|}
\hline & \multicolumn{11}{|c|}{ Spinal canal anteroposterior diameter (mm) } \\
\hline & C3 & C5 & C7 & $\mathrm{T1}$ & $\mathrm{T} 4$ & T8 & $\mathrm{T} 12$ & L1 & L3 & L5 & S1 \\
\hline All data $(n=83)$ & $14.6 \pm 1.12$ & $14.5 \pm 1.46$ & $15.2 \pm 1.75$ & $16.1 \pm 1.63$ & $15.3 \pm 1.21$ & $14.6 \pm 1.34$ & $16.7 \pm 1.20$ & $16.7 \pm 1.33$ & $15.7 \pm 1.90$ & $16.8 \pm 2.17$ & $17.4 \pm 2.29$ \\
\hline Women $(n=58)$ & $14.7 \pm 1.15$ & $14.6 \pm 1.49$ & $15.3 \pm 1.87$ & $16.1 \pm 1.61$ & $15.2 \pm 1.20$ & $14.7 \pm 1.38$ & $16.6 \pm 1.30$ & $16.9 \pm 1.31$ & $15.8 \pm 2.08$ & $17.0 \pm 2.07$ & $17.5 \pm 2.33$ \\
\hline Men $(n=25)$ & $14.8 \pm 1.07$ & $14.3 \pm 1.40$ & $15.2 \pm 1.48$ & $16.3 \pm 1.71$ & $15.5 \pm 1.26$ & $14.3 \pm 1.21$ & $16.8 \pm 0.94$ & $16.4 \pm 1.37$ & $15.3 \pm 1.59$ & $16.4 \pm 2.37$ & $17.1 \pm 2.21$ \\
\hline $\begin{array}{l}\text { Exposed canal after } \\
\text { laminectomy }\end{array}$ & $4.8 \pm 1.19$ & $4.7 \pm 1.24$ & $3.9 \pm 0.86$ & $3.3 \pm 0.89$ & 0 & - & - & - & - & - & - \\
\hline
\end{tabular}

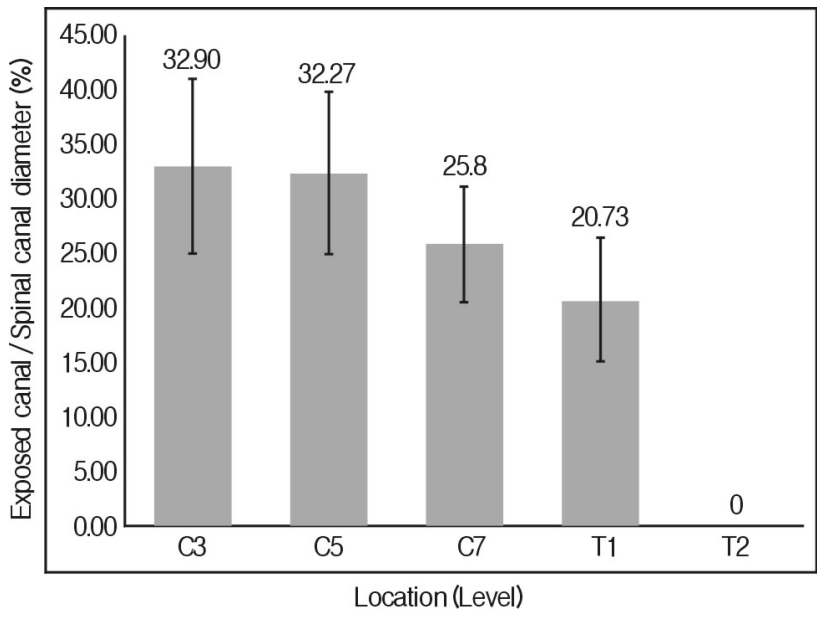

Fig. 8. The ratio of exposed spinal canal AP diameter above imaginary laminectomy line to spinal canal AP diameter, from C3 to $\mathrm{T} 2$.

\section{DISCUSSION}

The sagittal diameter of the spinal canal has clinical importance in traumatic, degenerative, and inflammatory conditions, and a small spinal canal diameter has been associated with an increased risk of injury ${ }^{15}$. Narrowing in the spinal canal can usually occur in the central part of the spinal canal or in the intervertebral foramen ${ }^{14)}$. To diagnose spinal stenosis, it is important to know the normal value of spinal canal cross-sectional area. However, due to its irregular shape, there are a lot of errors to measure the spinal cross-sectional area and it is almost impossible to measure the normal value of spinal cross-sectional area. Sagittal AP diameter of the spinal canal is the only parameter which could be statistically correlated with cross-sectional area, and it is accepted to use it as an indicator of the size of the spinal $\mathrm{canal}^{8)}$. There were many trials to define spinal stenosis by sagittal AP diameter of the spinal canal instead of cross-sectional area. Spinal stenosis has been defined by spinal canal AP diameter as follows: less than $12 \mathrm{~mm}$ for the cervical spine, less than $10 \mathrm{~mm}$ for the thoracic spine, and less than $12 \mathrm{~mm}$ for the lumbar spine $^{6,7,15,16,19)}$. To confirm spinal stenosis, the normal spinal canal AP diameters need to be established.

The cervical, thoracic, and lumbar spines have been studied with cadavers ${ }^{2,1416,19)}$. Sasaki et al. reported AP diameter of the cervical spine of a Japanese population as follows: $15.8 \mathrm{~mm}$ at $\mathrm{C} 3,15.3 \mathrm{~mm}$ at $\mathrm{C} 5$, and $15.9 \mathrm{~mm}$ at $\mathrm{C}^{19}$. They also noted the shortest AP diameter at $\mathrm{C} 3$ among cervical vertebrae. This result is very similar with our result. In another study by Michael et al., AP diameter of the cervical spine of a Western population was reported as follows: $14.3 \mathrm{~mm}$ at C3, $14.0 \mathrm{~mm}$ at $\mathrm{C}$, and $14.1 \mathrm{~mm}$ at $\mathrm{C} 7$. These results were shorter than our measurements. The difference may be attributed to the fact that we measured the spinal canal of modern people, while Michael et al. used human skeletons from the late nineteenth and early twentieth centuries.

Berry et al. reported AP diameters of $15.0 \mathrm{~mm}$ at T2, 16.6 $\mathrm{mm}$ at $\mathrm{T} 7,17.2 \mathrm{~mm}$ at $\mathrm{T} 12,17.2 \mathrm{~mm}$ at $\mathrm{L} 1,16.2 \mathrm{~mm}$ at $\mathrm{L} 3$, and $17.3 \mathrm{~mm}$ at $\mathrm{L} 5$, and the measurements for the thoracic spine were different from ours ${ }^{2}$. This discrepancy may also be attributed to the fact that they used human skeletons from the late nineteenth and early twentieth centuries and they studied only 30 vertebrae at each level. Lee et al. performed a morphometric study of the lumbar spinal canal in a Korean population ${ }^{14)}$. In their study, AP diameter gradually decreased from L1 $(15.4 \mathrm{~mm})$ to $\mathrm{L} 3(13.8 \mathrm{~mm})$ and then increased from L5 $(14.4 \mathrm{~mm})$ to the sacrum, and the pattern of changes in AP diameter was similar to our result. Because of restriction of specimens, cadaveric studies have a limitation in sample size. In fact, it is actually almost impossible to study adults without degeneration. As mentioned above, previous studies performed partial investigation of the spinal canal. Our study is the first anatomical study which examined the whole spine from cervical to sacrum in one person and in young adults without degenerative changes.

We also found that AP diameter of the exposed spinal canal above the imaginary laminectomy line was the longest at C3 $(4.8 \pm 1.2 \mathrm{~mm})$ and gradually decreased from $\mathrm{T} 1(3.3 \pm 0.9 \mathrm{~mm})$ to $\mathrm{T} 2(0 \mathrm{~mm})$. The ratio of exposed AP diameter to spinal AP diameter was also the largest at C3 (32.9\%). With this result, we can carefully infer that exposure of dural sac over lamina might be a factor of post-laminectomy membrane formation. 
Although PET/CT resolution is less than spine CT, anatomical study with PET/CT of the whole spine can be done with various ages and sizes at various population. However, $\mathrm{PET} / \mathrm{CT}$ has some issues, and radiation is the main one ${ }^{18)}$. Recently, we did not used PET/CT for analysis in cancer screening due to radiation exposure. However, the data analyzed in this study were of patients who underwent PET/CT for internal organ cancer, not for the spine or nervous system, to rule out metastasis. For these patients, PET/CT is essential ${ }^{1,3,9,11,12)}$.

In conclusion, axial bone-window CT obtained from PET/ CT may be useful to study anatomical bony structure of spine. Spinal AP diameter was the shortest at the mid- cervical vertebra and increased in the upper thoracic area. At the thoracic vertebrae, this paper provides a comprehensive review to morphometric survey of spinal canal in whole level in Korean young adult. AP diameter is decreased from the upper thoracic vertebra to the mid- thoracic vertebra (T8) and similar pattern was identified in lumbar region. T1, there was no dural sac that would be exposed after laminectomy. This means that restenosis by post-laminectomy membrane formation can occur above T1.

Conflict of interest: The authors declare that they have no conflict of interest.

\section{REFERENCES}

1. Alberini JL, Edeline V, Giraudet AL, Champion L, Paulmier B, Madar O, et al: Single photon emission tomography/ computed tomography (spet/ct) and positron emission tomography/computed tomography (pet/ct) to image cancer. J Surg Oncol 103: 602-606, 2011

2. Berry JL, Moran JM, Berg WS, Steffee AD: A morphometric study of human lumbar and selected thoracic vertebrae. Spine (Phila Pa 1976) 12:362-367, 1987

3. Castellucci P, Fuccio C, Marzola MC, Al-Nahhas A, Rubello D, Fanti S: Prostate-specific antigen kinetics and choline pet/ct in patients with biochemical relapse after primary treatment for prostate cancer. Nucl Med Commun 32:475-478, 2011

4. Cerisoli M, Vernizzi E, Giulioni M: Cervical spine changes following laminectomy. Clinico-radiological study. J Neurosurg Sci 24:63-70, 1980

5. Cheung KM, Ruan D, Chan FL, Fang D: Computed tomographic osteometry of asian lumbar pedicles. Spine (Phila Pa 1976) 19:1495-1498, 1994

6. Epstein NE, Schwall G: Thoracic spinal stenosis: diagnostic and treatment challenges. J Spinal Disord 7:259-269, 1994

7. Geisser ME, Haig AJ, Tong HC, Yamakawa KS, Quint DJ, Hoff
JT, et al: Spinal canal size and clinical symptoms among persons diagnosed with lumbar spinal stenosis. Clin J Pain 23:780-785, 2007

8. Gepstein R, Folman Y, Sagiv P, Ben David Y, Hallel T: Does the anteroposterior diameter of the bony spinal canal reflect its size? An anatomical study. Surg Radiol Anat 13:289-291, 1991

9. Haug AR, Bartenstein P: [positron emission tomography (pet) and pet/ct for guiding therapy in oncology]. Dtsch Med Wochenschr1 36:468-472, 2011

10. Heller JG, Edwards CC $2^{\text {nd }}$, Murakami H, Rodts GE: Laminoplasty versus laminectomy and fusion for multilevel cervical myelopathy: an independent matched cohort analysis. Spine (Phila PA 1976) 26:1330-1336, 2001

11. Kim YS, Kim SJ, Kim YK, Kim IJ, Kim YD, Lee MK: Prediction of survival and cancer recurrence using $\mathrm{f}-18 \mathrm{fdg}$ pet/ct in patients with surgically resected early stage (stage $i$ and ii) nonsmall cell lung cancer. Neoplasma 58:245-250, 2011

12. Kitajima K, Murakami K, Kaji Y, Sakamoto S, Sugimura K: Established, emerging and future applications of fdg-pet/ct in the uterine cancer. Clin Radiol 66:297-307, 2011

13. Kretzer RM, Chaput C, Sciubba DM, Garonzik IM, Jallo GI, McAfee PC, et al: A computed tomography-based morphometric study of thoracic pedicle anatomy in a random united states trauma population. J Neurosurg Spine 14:235-243, 2011

14. Lee HM, Kim NH, Kim HJ, Chung IH: Morphometric study of the lumbar spinal canal in the korean population. Spine (Phila Pa 1976) 20:1679-1684, 1995

15. Lee MJ, Cassinelli EH, Riew KD: Prevalence of cervical spine stenosis. Anatomic study in cadavers. J Bone Joint Surg Am 89: 376-380, 2007

16. Lee MJ, Garcia R, Cassinelli EH, Furey C, Riew KD: Tandem stenosis: acadaveric study in osseous morphology. Spine J 8: 1003-1006, 2008

17. Lee SH, Tsang YS, Chung SE, Shin SW, Kang HY: Height and depth of the cervical disc space in normal korean adults. Korean J Spine 11:121-126, 2004

18. Morimoto T, Ohtsuka H, Sakaki T, Kawaguchi M: Postlamine ctomy cervical spinal cord compression demonstrated by dyna mic magnetic resonance imaging. Case report. J Neurosurg 88: 155-157, 1998

19. Murano T, Minamimoto R, Senda M, Uno K, Jinnouchi S, Fukuda $\mathrm{H}$, et al: Radiation exposure and risk-benefit analysis in cancer screening using fdg-pet: results of a japanese nationwide survey. Ann Nucl Med, 2011

20. Sasaki T, Kadoya S, lizuka H: Roentgenological study of the sagittal diameter of the cervical spinal canal in normal adult japanese. Neurol Med Chir 38:83-88, 1998

21. Vara CS, Thompson GH: A cadaveric examination of pediatric cervical pedicle morphology. Spine (Phila Pa 1976) 31: 1107-1112, 2006

22. Wu LP, Li YK, Li YM, Zhang YQ, Zhong SZ: Variable morphology of the sacrum in a chinese population. ClinAna 22:619626,2009 